\title{
Stability analysis for laminates with general anisotropy using boundary elements
}

\author{
S. Syngellakis \\ Wessex Institute of Technology, UK
}

\begin{abstract}
A numerical analysis, based on the boundary element method, is proposed for predicting the buckling behaviour of asymmetrically laminated, anisotropic plates. The modelling accounts for the coupling between bending and in-plane extension, which characterises the constitutive relations of such plates. The problem is formulated in terms of the in-plane displacements and transverse deflection using the fundamental solutions of the uncoupled in-plane extension and bending problems governing the behaviour of symmetrically laminated plates. As a consequence, irreducible domain integrals appear in the derived integral equations; this necessitates the adoption of domain in-plane strains and curvatures as unknown variables and the concomitant derivation of additional integral equations. Thus both problems governing the pre-buckling state as well as the buckling mode and critical load comprise consistent systems of equations. The high-order singularity of certain domain integrals is addressed and a comparison is made between the present analysis and that formulated in terms of the stress function and transverse deflection.

Keywords: laminates, bending-stretching coupling, buckling, in-plane displacements, boundary elements.
\end{abstract}

\section{Introduction}

General laminates, comprising layers with orthotropic axes of symmetry in various orientations, exhibit a special type of anisotropy characterised by coupling between in-plane extension and flexure. Early closed form solutions for transverse deflection, buckling load and natural frequencies were obtained in the special cases of the cross-ply and angle-ply laminates [1]. The use of Fourier series [2] allowed the investigation of the effect of various combinations of 
boundary conditions on the flexure, buckling and free vibration results [3]. The effect of coupling on the buckling load and post-buckling stiffness was studied for laminates of specific anisotropy [4].

The boundary element method (BEM) has been extensively applied to the uncoupled in-plane extension and flexural problems representing the behaviour of balanced, symmetrically laminated plates [5]. Recently, the BEM methodology was extended to the general anisotropy of the asymmetrically laminated plates; the developed formulation was based on fundamental solutions that account for constitutive bending-stretching coupling [6].

Early attempts at buckling analysis of anisotropic plates by BEM had to consider bending-stretching coupling arising from the geometric non-linearity of the problem. The BEM methodology already applied to isotropic plate buckling was extended to rectangular orthotropic plates under uniform in-plane loading [7]; the term arising from stretching-bending coupling was represented as a fictitious lateral load which was modelled over the domain and related to the other problem variables through an additional integral equation. An alternative formulation was subsequently proposed whereby the irreducible domain integral depended on transverse deflection, which was adopted as the domain unknown variable [8]. This approach was complemented with a mathematically similar procedure for the in-plane extension formulated in terms of the stress function; this generated accurate buckling load predictions even in cases of non-uniform in-plane loading [9]. The same solution methodology was extended to non-linear buckling through an incremental and iterative procedure [10]. A BEM solution to the buckling of general, unbalanced laminates was recently developed in terms of the stress function and transverse deflection using for both variables the same fundamental solution associated with the forth order differential operator governing anisotropic plate flexure [11].

In this paper, the formulated BEM buckling analysis also accounts for the general form of plate anisotropy encountered in unbalanced laminates. In contrast to the earlier approach however, the in-plane extension problem is here formulated in terms of the mid-plane displacements. This allows greater flexibility in specifying in-plane boundary conditions but requires a fundamental solution mathematically different from that associated with transverse deflection. The integrability of certain integrals is examined in view of the high-order singularity of the respective kernels arising from that fundamental solution. Although focusing on the solution of the linear buckling problem, the formulation can be extended to the prediction of the nonlinear, post-buckling behaviour of laminates under in-plane loading as well as the large bending deformation under any combination of domain body and transverse forces as well as other types of boundary loading.

\section{Non-linear laminate theory}

A laminate is made by stacking layers, unidirectionally reinforced at different fibre orientations. Its effective properties vary with the orientation, thickness, and stacking sequence of the individual layers. An anisotropic constitutive theory 
for a laminate is derived assuming that the layers (laminate or plies) are perfectly bonded, the material in each layer is homogeneous and can be isotropic, orthotropic, or transversely isotropic with known effective properties, each layer is in a state of plane stress and the Kirchhoff assumptions for bending and stretching of plates are valid.

Using tensor notation and referring to a Cartesian frame of reference $x_{1}, x_{2}$, the membrane forces $N_{\alpha \beta}$ and bending moments $M_{\alpha \beta}$ are, according to the above theory, related to the mid-plane strains $\varepsilon_{\alpha \beta}$ and curvatures $\kappa_{\alpha \beta}$ by

$$
\begin{aligned}
& N_{\alpha \beta}=A_{\alpha \beta \gamma \delta} \varepsilon_{\gamma \delta}+B_{\alpha \beta \gamma \delta} \kappa_{\gamma \delta} \\
& M_{\alpha \beta}=B_{\alpha \beta \gamma \delta} \varepsilon_{\gamma \delta}+D_{\alpha \beta \gamma \delta} \kappa_{\gamma \delta}
\end{aligned}
$$

where $A_{\alpha \beta \gamma \delta}$ are the extensional stiffness coefficients, $D_{\alpha \beta \gamma \delta}$ are the flexural stiffness coefficients and $B_{\alpha \beta \gamma \delta}$ are the bending-stretching coupling coefficients with the range of all Greek indices from 1 to 2 and the summation convention over repeated indices. According to von Kármán's non-linear theory for thin elastic plates [12], the mid-plane strains $\varepsilon_{\alpha \beta}$ and curvatures $\kappa_{\alpha \beta}$ are given in terms of the in-plane displacements $u_{\alpha}$ and transverse deflection $w$ by

$$
\begin{gathered}
\varepsilon_{\alpha \beta}=1 / 2\left(u_{\alpha, \beta}+u_{\beta, \alpha}+w,{ }_{\alpha} w,{ }_{\beta}\right) \\
\kappa_{\alpha \beta}=-w,{ }_{\alpha \beta}
\end{gathered}
$$

where a comma followed by a lower index indicates differentiation with respect to the corresponding co-ordinate. The governing field equations and boundary conditions can be obtained by minimising the potential energy functional $\Pi$ given by

$$
\Pi=1 / 2 \int_{\Omega}\left(N_{\alpha \beta} \varrho_{\alpha \beta}+M_{\alpha \beta} \kappa_{\alpha \beta}\right) \mathrm{d}-\int_{\Omega} q w \mathbb{Q}-\int_{\Gamma} \tilde{p}_{\alpha} u_{\alpha} \mathrm{d} s
$$

where $q$ is the transverse pressure, $\tilde{p}_{\alpha}$ the prescribed edge traction, $\Omega$ the plate domain bounded by the contour $\Gamma$ and $s\left(x_{1}, x_{2}\right)$ the path variable along $\Gamma$, which is assumed smooth apart from a finite number of corner points. The potential energy functional $\Pi$ can be expanded to include more "external work" terms arising, for instance, from in-plane body forces or other types of edge loading. Along the plate boundary, the state of stress is described by traction $p_{\alpha}$, bending moment $M_{n}$, twisting moment $M_{n s}$ and shear force $V_{n}$ which are given by

$$
\begin{gathered}
p_{\alpha}=n_{\beta} N_{\alpha \beta} \\
M_{n}=n_{\alpha} n_{\beta} M_{\alpha \beta} \\
M_{n s}=s_{\alpha} n_{\beta} M_{\alpha \beta} \\
V_{n}=n_{\alpha} M_{\alpha \beta, \beta}+\frac{\partial M_{n s}}{\partial s}
\end{gathered}
$$

while force $C_{j}$ represents the discontinuity jump of $M_{n s}$ at corner $j$. Unit vectors $\mathbf{n}\left(n_{1}, n_{2}\right)$ and $\mathbf{s}\left(s_{1}, s_{2}\right)$ are, respectively, normal and tangent to $\Gamma$. A plate schematic illustrating the adopted notation is given in fig. 1.

Substituting the variations of $\varepsilon_{\alpha \beta}$ and $\kappa_{\alpha \beta}$, obtained, respectively, from eqns (3) and (4), into the condition $\delta \Pi=0$, performing integrations by parts and applying repeatedly Green's theorem yields the field equations

$$
\begin{gathered}
N_{\alpha \beta, \beta}=0 \\
M_{\alpha \beta, \alpha \beta}+N_{\alpha \beta} w, \alpha \beta \\
+q=0
\end{gathered}
$$


over the domain $\Omega$, the boundary conditions

$$
\begin{gathered}
\text { either } p_{\alpha}=\tilde{p}_{\alpha} \text { or } u_{\alpha}=0 \\
\text { either } M_{n}=0 \text { or } \frac{\partial w}{\partial n}=0 \\
\text { either } V_{n}+p_{\alpha} w,_{\alpha}=0 \text { or } w=0
\end{gathered}
$$

along $\Gamma$ and

at any corner $j$ with path co-ordinate $s_{j}$.

$$
\text { either } C_{j}=0 \text { or } w_{j}=w\left(s_{j}\right)=0
$$

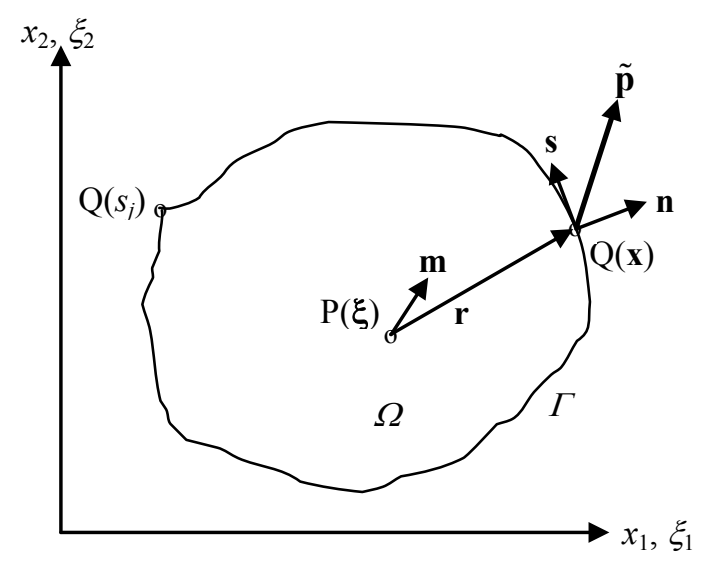

Figure 1: $\quad$ Laminate plate of arbitrary shape under edge traction $\tilde{\mathbf{p}}$.

\section{Pre-buckling state}

Instability may be caused by factored edge traction $\lambda \tilde{p}_{\alpha}^{0}$ where $\tilde{p}_{\alpha}^{0}$ is a low reference loading. It is assumed that, under $\tilde{p}_{\alpha}^{0}$, a stable pre-buckling state exists, characterised by stress resultants $N_{\alpha \beta}^{0}, M_{\alpha \beta}^{0}$ and deformation components $u_{\alpha}^{0}, w^{0}$, which should satisfy the field equations and boundary conditions presented in the previous section. In the special case of balanced, linearly deformed laminates, the general theory uncouples into two classical anisotropic problems, namely inplane extension and plate bending, for which the respective fundamental solutions $u_{\lambda \gamma}^{*}$ and $w_{\lambda}^{*}$ have been derived. The former can be used to obtain the BEM solution of the membrane stress problem according to a procedure similar to that applied to two-dimensional elasticity [13]; the latter leads to the BEM solution of the anisotropic plate problem $[5,14]$.

The fundamental solution for in-plane extension satisfies

$$
A_{\alpha \beta \gamma \delta} u_{\lambda \gamma}^{*},_{\beta \delta}+\delta_{\alpha \lambda} \delta(\mathbf{x}-\xi)=0
$$


where $\mathbf{x}\left(x_{1}, x_{2}\right)$ is the field point, $\xi\left(\xi_{1}, \xi_{2}\right)$ the source point, $\delta_{\alpha \beta}$ the Kronecker delta and $\delta(\mathbf{x}-\xi)$ the Dirac delta function. Various versions for $u_{\lambda \gamma}^{*}$ can be found in earlier publications describing BEM formulations for two dimensional orthotropic or anisotropic elasticity. In the context of the present analysis, $u_{\lambda \gamma}^{*}$ was derived, using Fourier transforms, in a rather compact form, which is given in the appendix.

The fundamental solution for plate bending satisfies

$$
D_{\alpha \beta \gamma \delta} w_{\lambda}^{*},_{\alpha \beta \gamma \delta}=\delta_{\lambda}(\mathbf{x}-\xi)
$$

where

$$
\delta_{1}(\mathbf{x}-\xi)=\delta(\mathbf{x}-\xi) \text { and } \delta_{2}(\mathbf{x}-\xi)=\frac{\partial \delta(\mathbf{x}-\xi)}{\partial m(\xi)}
$$

The two infinite plate deflections at a field point $\mathrm{Q}(\mathbf{x})$ given by eqn (17) are interpreted as [12]: $w_{1}^{*}$ due to a transverse unit point force at the source point $\mathrm{P}(\xi), w_{2}^{*}$ due to a unit moment at $\mathrm{P}$ about the direction normal to unit vector $\mathbf{m}$ as schematically shown in fig. 1 . Compact expressions for $w_{\lambda}^{*}$ and the other kernels arising from them are given in an earlier article on a BEM application to the plate bending problem [5]. For future reference, the expressions for the first fundamental solution and the respective curvatures are given in the appendix.

Integral equations for the coupled stretching-bending problem are here derived by taking advantage of the symmetry of both $A_{\alpha \beta \gamma \delta}$ and $D_{\alpha \beta \gamma \delta}$ tensors. It is thus possible to formulate the reciprocity relations

$$
\begin{gathered}
\int_{\Omega} A_{\alpha \beta \gamma \delta} u_{\alpha}^{0},_{\beta} u_{\lambda \gamma, \delta}^{*} \mathrm{~d} \Omega=\int_{\Omega} A_{\alpha \beta \gamma \delta} u_{\lambda \alpha}^{*},_{\beta} u_{\gamma}^{0},_{\delta} \mathrm{d} \Omega \\
\int_{\Omega} D_{\alpha \beta \gamma \delta} w^{0},_{\alpha \beta} w_{\lambda,{ }_{\gamma \delta}} \mathrm{d} \Omega=\int_{\Omega} D_{\alpha \beta \gamma \delta} w_{\lambda, \alpha \beta}^{*} w^{0},_{\gamma \delta} \mathrm{d} \Omega
\end{gathered}
$$

Taking into account the constitutive relations for both the actual, coupled nonlinear as well as the fundamental linear states, eqns (18) and (19) are transformed to

$$
\begin{gathered}
k u_{\lambda}^{0}(\xi)=I_{b}^{u}\left(u_{\alpha}^{0}, u_{\lambda \alpha}^{*}\right)-I_{d}^{c}\left(\varepsilon_{\lambda \alpha \beta}^{*}, \kappa_{\gamma \delta}^{0}\right)-I_{d}^{n}\left(\varepsilon_{\lambda \alpha \beta}^{*}, w^{0},_{\alpha}\right) \\
k w_{\lambda}^{0}(\xi)=I_{b}^{w}\left(w^{0}, w_{\lambda}^{*}\right)+J^{w}\left(w^{0}, w_{\lambda}^{*}\right)+\int_{\Omega} N_{\alpha \beta}^{0} w^{0},_{\alpha \beta} w_{\lambda}^{*} \mathrm{~d} \Omega-I_{d}^{c}\left(\varepsilon_{\alpha \beta}^{0}, \kappa_{\lambda \gamma \delta}^{*}\right)
\end{gathered}
$$

where $k=1$ or $1 / 2$, depending on whether $\mathrm{P}(\xi)$ lies within $\Omega$ or belongs to smooth portions of $\Gamma$, respectively, and

$$
w_{1}^{0}=w^{0}, \quad w_{2}^{0}=\frac{\partial w^{0}}{\partial n}
$$

The right-hand sides of eqns (20) and (21) comprise the boundary and domain integrals as well as a jump term which are defined by the equations

$$
\begin{gathered}
I_{b}^{u}\left(u_{\alpha}, u_{\alpha}^{\prime}\right)=\int_{\Gamma}\left(p_{\alpha}\left(u_{\beta}\right) u_{\alpha}^{\prime}-p_{\alpha}\left(u_{\beta}^{\prime}\right) u_{\alpha}\right) \mathrm{d} s \\
I_{b}^{w}\left(w, w^{\prime}\right)=\int_{\Gamma}\left[V_{n}(w) w^{\prime}-M_{n}(w) \frac{\partial w^{\prime}}{\partial n}+M_{n}\left(w^{\prime}\right) \frac{\partial w}{\partial n}-V_{n}\left(w^{\prime}\right) w\right] \mathrm{d} s
\end{gathered}
$$




$$
\begin{gathered}
I_{d}^{c}\left(\varepsilon_{\alpha \beta}, \kappa_{\alpha \beta}\right)=\int_{\Omega} B_{\alpha \beta \gamma \delta} \varepsilon_{\alpha \beta} \kappa_{\gamma \delta} \mathrm{d} \Omega, \\
I_{d}^{n}\left(\varepsilon_{\alpha \beta}, w,_{\alpha}\right)=\frac{1}{2} \int_{\Omega} A_{\alpha \beta \gamma \delta} \varepsilon_{\alpha \beta} w,_{\gamma} w,_{\delta} \mathrm{d} \Omega \\
J^{w}\left(w, w^{\prime}\right)=\sum_{j=1}^{K}\left[C_{j}(w) w_{j}^{\prime}-C_{j}\left(w^{\prime}\right) w_{j}\right]
\end{gathered}
$$

where $K$ is the number of corner points along $\Gamma$. It should be noted that, because of constitutive stretching-bending coupling, $p_{\alpha}^{0}\left(u_{\alpha}^{0}\right)$ also depends on $w^{0}$; similarly, the boundary variables $M_{n}^{0}\left(w^{0}\right), M_{n s}^{0}\left(w^{0}\right), V_{n}^{0}\left(w^{0}\right)$ also depend on $u_{\alpha}^{0}$. The domain integral $I_{d}^{c}\left(\varepsilon_{\alpha \beta}^{0}, \kappa_{\lambda \gamma \delta}^{*}\right)$, arising from constitutive coupling, incorporates also geometric nonlinearity through $\varepsilon_{\alpha \beta}^{0}$.

The pair of integral equations (20) and (21) contains domain integrals depending on the unknown mid-plane strains, curvatures and deflection gradients. A numerical load-stepping scheme can be devised for their solution, which would describe both the pre-buckling state as well as the general nonlinear response. For a high value of $\lambda$, the latter represents the non-linear buckling behaviour of the plate. The inclusion of body and transverse forces as well as other types of boundary loading in the formulation would lead to the solution of the general coupled non-linear bending problem.

In the present paper, the focus is on bifurcation buckling due to in-plane compressive forces. It is therefore reasonable to assume that all components of pre-buckling deformation are small and, as a consequence, all domain integrals arising from geometric non-linearity can be ignored. This means that the only irreducible domain integrals remaining in eqns (20) and (21) are those arising from constitutive stretching-bending coupling, namely,

$$
\begin{aligned}
& I_{d}^{c}\left(\varepsilon_{\lambda \alpha \beta}^{*}, \kappa_{\gamma \delta}^{0}\right)=\int_{\Omega} B_{\alpha \beta \gamma \delta} \varepsilon_{\lambda \alpha \beta}^{*} \kappa_{\gamma \delta}^{0} \mathrm{~d} \Omega=-\int_{\Omega} B_{\alpha \beta \gamma \delta} u_{\lambda \alpha}^{*},_{\beta} w^{0},_{\gamma \delta} \mathrm{d} \Omega \\
& I_{d}^{c}\left(\varepsilon_{\alpha \beta}^{0}, \kappa_{\lambda \gamma \delta}^{*}\right)=\int_{\Omega} B_{\alpha \beta \gamma \delta} \varepsilon_{\alpha \beta}^{0} \kappa_{\lambda \gamma \delta}^{*} \mathrm{~d} \Omega=-\int_{\Omega} B_{\alpha \beta \gamma \delta} u_{\alpha}^{0},_{\beta} w_{\lambda,{ }_{\gamma \delta}}^{*} \mathrm{~d} \Omega
\end{aligned}
$$

A possible way of generating a numerical solution of the coupled linear problem would be to derive additional integral equations for the domain midplane strains and curvatures. For this purpose, eqns (20) and (21) are first applied with the source point in the domain $(k=1)$ and then differentiated with respect to $\xi_{\alpha}$ to give

$$
\begin{gathered}
u_{\mu,,_{\nu}}^{0}(\xi)=I_{b}^{u}\left(u_{\alpha}^{0}, u_{\alpha \mu}^{*},_{\nu}\right)-\frac{\partial}{\partial \xi_{v}} \int_{\Omega} B_{\alpha \beta \gamma \delta} u_{\alpha \mu}^{*},_{\beta} \kappa_{\gamma \delta}^{0} \mathrm{~d} \Omega \\
w^{0},_{\mu \nu}(\xi)=I_{b}^{w}\left(w^{0}, w_{1}^{*},_{\mu \nu}\right)+J^{w}\left(w^{0}, w_{1}^{*},_{\mu \nu}\right)+\frac{\partial^{2}}{\partial \xi_{\mu} \partial \xi_{v}} \int_{\Omega}\left(B_{\gamma \delta \alpha \beta} \varepsilon_{\gamma \delta}^{0}\right) w_{1}^{*},_{\alpha \beta} \mathrm{d} \Omega
\end{gathered}
$$

As indicated by eqn (41) in the appendix, the kernel $\varepsilon_{\lambda \gamma \delta}^{*}$ is of order $\mathrm{O}\left(r^{-1}\right)$ and therefore integrable in $\Omega$. However, the strong singularity of $\varepsilon_{\lambda \gamma \delta}^{*},{ }_{\nu}$ does not allow differentiation under the domain integral of eqn (27). A similar observation 
applies to the domain integral of eqn (28). Although the kernels $\kappa_{1 \gamma \delta}^{*}$ are regular, according to eqn (43) in the appendix, their second derivatives are also of order $\mathrm{O}\left(r^{-2}\right)$ and therefore differentiation under the respective is integral is not allowed. The evaluation of the derivatives of these domain integrals can be achieved in the manner adopted in a similar BEM approach to anisotropic elasticity analysis [15], which is based on the concept of convected differentiation of a singular integral [16]. The process leads to the derivation of a convective term from each derivative and the domain integrals

$$
\begin{gathered}
\int_{\Omega} B_{\alpha \beta \gamma \delta} \frac{\partial u_{\alpha \mu}^{*}, \beta}{\partial \xi_{v}} \kappa_{\gamma \delta}^{0} \mathrm{~d} \Omega=-\int_{\Omega} B_{\alpha \beta \gamma \delta} u_{\alpha \mu}^{*},{ }_{\beta} \kappa_{\gamma \delta}^{0} \mathrm{~d} \Omega \\
\int_{\Omega}\left(B_{\gamma \delta \alpha \beta} \varepsilon_{\gamma \delta}^{0}\right) \frac{\partial^{2} w_{1}^{*}, \alpha \beta}{\partial \xi_{\mu} \partial \xi_{v}} \mathrm{~d} \Omega=\int_{\Omega}\left(B_{\gamma \delta \alpha \beta} \varepsilon_{\gamma \delta}^{0}\right) w_{1}^{*},{ }_{\alpha \beta \mu \nu} \mathrm{d} \Omega
\end{gathered}
$$

whose kernels are strongly singular, that is, of order $\mathrm{O}\left(r^{-2}\right)$. The Cauchy principal values of these integrals can be evaluated by a process that divides each of them into a regular and a contour integral [17]. The latter is conveniently evaluated along the boundary of a domain cell encompassing the source point.

In addition to boundary modelling, domain modelling for the mid-plane strains and curvatures is necessary for the generation of a consistent system of algebraic equations through the application of eqns (20) and (21) with the source point load at all boundary nodes and eqns (27) and (28) with the source point load at all domain nodes. Such schemes using discontinuous boundary elements and domain cells have been described in previous BEM formulations of laminate bending and buckling problems $[5,9]$.

\section{Critical equilibrium state}

The pre-buckling state of equilibrium becomes unstable at a certain intensity of the load factor $\lambda$. Then a second stable equilibrium state exists, which can be asymptotically identified through the solution of a series of linear variational principles [18]. The critical load factor $\lambda_{c}$ and the buckling mode $u_{\alpha}^{1}, w^{1}$ can be determined from the first such variational equation

$$
\int_{\Omega}\left(N_{\alpha \beta}^{1} \delta u_{\alpha},,_{\beta}-M_{\alpha \beta}^{1} \delta w_{\alpha \beta}\right) \mathrm{d} \Omega+\lambda_{c} \int_{\Omega}\left(N_{\alpha \beta}^{1} w^{0},_{\alpha} \delta w_{\beta}+N_{\alpha \beta}^{0} w^{1},_{\alpha} \delta w_{\beta}\right) \mathrm{d} \Omega=0
$$

where $N_{\alpha \beta}^{1}$ and $M_{\alpha \beta}^{1}$ are linearly related to the corresponding strains and curvatures according to eqns (1) and (2). Because of the assumption of linear pre-buckling state, the relation between $\varepsilon_{\alpha \beta}^{1}$ and $u_{\alpha}^{1}$ is linear as well. By a process similar to that applied to the variation of $\Pi$ in section 2, integral eqn (29) leads to the field equations

$$
\begin{gathered}
N_{\alpha \beta}^{1},_{\beta}=0 \\
M_{\alpha \beta}^{1},_{\alpha \beta}+\lambda_{c}\left(N_{\alpha \beta}^{1} w^{0}{ }_{\alpha \beta}+N_{\alpha \beta}^{0} w^{1}{ }_{\alpha \beta}\right)=0
\end{gathered}
$$

over the domain $\Omega$ and the boundary conditions, 


$$
\begin{gathered}
\text { either } p_{\alpha}^{1}=0 \text { or } u_{\alpha}^{1}=0 \\
\text { either } M_{n}^{1}=0 \text { or } \frac{\partial w^{1}}{\partial n}=0 \\
\text { either } V_{n}^{1}+\lambda_{c} p_{\alpha}^{0} w^{1},_{\alpha}=0 \text { or } w^{1}=0
\end{gathered}
$$

on $\Gamma$ and

$$
\text { either } C^{1}\left(s_{j}\right)=0 \text { or } w^{1}\left(s_{j}\right)=0
$$

at the corner $j$, where all boundary variables at the critical equilibrium state are related to the respective domain variables according to eqns (6)-(9). A BEMbased procedure is formulated for the numerical solution of the boundary value problem described by eqns (30)-(35).

The proposed formulation is again based on reciprocity relations similar to those given by eqns (18) and (19) where the pre-buckling displacements $\left(u_{\alpha}^{0}, w^{0}\right)$ are replaced by the buckling mode displacements $\left(u_{\alpha}^{1}, w^{1}\right)$. Accounting for the field equations (30) and (31) satisfied by the buckling mode eliminates some domain integrals generated by the application of Green's theorem. As with the pre-buckling solution however, irreducible domain integrals remain in the derived integral equations due to the use of fundamental solutions $u_{\lambda \alpha}^{*}$ and $w_{\lambda}^{*}$ for the uncoupled in-plane extension and bending problems, respectively. The form of the resulting integral equations is:

$$
\begin{gathered}
k u_{\lambda}^{1}(\xi)=I_{b}^{u}\left(u_{\alpha}^{1}, u_{\lambda \alpha}^{*}\right)-I_{d}^{c}\left(\varepsilon_{\lambda \alpha \beta}^{*}, \kappa_{\gamma \delta}^{1}\right) \\
k w_{\lambda}^{1}(\xi)=I_{b}^{w}\left(w^{1}, w_{\lambda}^{*}\right)+J^{w}\left(w^{1}, w_{\lambda}^{*}\right) \\
+\lambda_{c} \int_{\Omega}\left(N_{\alpha \beta}^{1} w^{0},_{\alpha \beta}+N_{\alpha \beta}^{0} w^{1},_{\alpha \beta}\right) w_{\lambda}^{*} \mathrm{~d} \Omega-I_{d}^{c}\left(\varepsilon_{\gamma \delta}^{1}, \kappa_{\alpha \beta \lambda}^{*}\right)
\end{gathered}
$$

where

$$
w_{1}^{1}=w^{1}, \quad w_{2}^{1}=\frac{\partial w^{1}}{\partial n}
$$

and the boundary integrals, domain integrals and the jump term are defined according to eqns (22)-(26).

As with the pre-buckling state, the standard BEM modelling process is not applicable also in the case of the buckling problem, due to the presence of several domain integrals in eqns (36) and (37) depending on the unknown inplane strains and stress resultants as well as curvatures. This mathematical difficulty is again overcome by introducing modelling for the unknown domain strains and curvatures and the additional integral equations

$$
\begin{gathered}
u_{\mu,,_{\nu}}^{1}(\xi)=I_{b}^{u}\left(u_{\alpha}^{1}, u_{\alpha \mu}^{*},_{\nu}\right)-\frac{\partial}{\partial \xi_{\nu}} \int_{\Omega} B_{\alpha \beta \gamma \delta} u_{\alpha \mu}^{*},{ }_{\beta} \kappa_{\gamma \delta}^{1} \mathrm{~d} \Omega \\
w^{1},_{\mu \nu}(\xi)=I_{b}^{w}\left(w^{1}, w_{1}^{*},{ }_{\mu \nu}\right)+J^{w}\left(w^{1}, w_{1}^{*},_{\mu \nu}\right)
\end{gathered}
$$




$$
+\lambda_{c} \int_{\Omega}\left(N_{\alpha \beta}^{1} w^{0},_{\alpha \beta}+N_{\alpha \beta}^{0} w^{1},_{\alpha \beta}\right) w_{1}^{*},_{\mu \nu} \mathrm{d} \Omega+\frac{\partial^{2}}{\partial \xi_{\mu} \partial \xi_{v}} \int_{\Omega} B_{\alpha \beta \gamma \delta} \varepsilon_{\gamma \delta}^{1} w_{1}^{*},_{\alpha \beta} \mathrm{d} \Omega
$$

obtained by differentiating both sides of eqns (36) and (37) with respect to $\xi_{\alpha}$ with the source point in the domain $(k=1)$.

The kernel of the domain integral arising from geometric non-linearity has logarithmic singularity, it is therefore integrable. Regarding the derivatives of the other two domain integrals arising from constitutive stretching-bending coupling, their evaluation process is the same as that described for similar terms appearing in eqns (27) and (28). Introducing the same boundary and domain modelling schemes as those employed for the pre-buckling solution, eqns (36)-(39) are transformed into systems of linear algebraic equations, which can be re-arranged into a classical eigenvalue problem. The lowest eigenvalue would be the critical load factor $\lambda_{c}$.

\section{Discussion}

The feasibility of a BEM-based approach for the buckling analysis of general laminates was demonstrated in the previous sections. The mathematical complication arising from stretching-bending coupling is addressed through additional integral equations and domain modelling. A similar such procedure was recently proposed [11] characterised by elegance and economy because it comprised two mathematically identical problems requiring a common fundamental solution. Since it was developed in terms of the stress function, it had the disadvantage of limited options for specifying in-plane constraints or loading. Although a useful tool for a linear critical load analysis, it would be difficult to extend it to non-linear coupled bending-stretching problems.

It was pointed out in the introduction that, in comparison with the BEM analysis formulated in terms of the stress function, the present method of solution, formulated in terms of mid-plane displacements, has the advantage of admitting directly any combination of in-plane boundary constraint or loading. It can thus be easily extended to the solution of more complex non-linear problems. On the other hand, more programming effort is required because of the use of two different fundamental solutions for the in-plane extension and bending problems. It is also computationally more demanding due to the presence of additional domain integrals, especially in the case of the general non-linear problem.

The final test regarding the efficiency, reliability and versatility of both BEM schemes would come with their computer implementation. The development of validated computer codes and their application to a wide range of cases would provide a more solid basis for their comparison.

Domain modelling with the concomitant introduction of additional unknowns enlarges considerably the size of the problem and computational time and effort. An important future research direction would therefore be towards devising alternative modelling schemes whereby either domain unknown are reduced or 
domain meshing is avoided or domain integrals are converted to boundary ones dual reciprocity techniques.

\section{Appendix}

In the context of the in-plane extension problem, the 4th-order tensors $\Delta_{\alpha \beta \gamma \delta}$ and $\Lambda_{\alpha \beta \gamma \delta}$ are defined by

$$
\begin{gathered}
\Delta_{\alpha \beta \gamma \delta}=e_{\alpha \lambda} e_{\beta \kappa} A_{\kappa \gamma \lambda \delta} \\
\Lambda_{\alpha \beta \gamma \delta}=\frac{1}{2} e_{\kappa \mu} e_{\lambda v} A_{\kappa \alpha \lambda \beta} A_{\mu \gamma \nu \delta}
\end{gathered}
$$

where $e_{\alpha \beta}$ is the two-dimensional special skew-symmetric tensor $\left(e_{11}=e_{22}=0\right.$, $\left.e_{12}=-e_{21}=1\right)$. Then, the quadratic functions $F_{\alpha \beta}(\omega)$ are defined by

$$
F_{\alpha \beta}(\omega)=\frac{\Delta_{\alpha \beta \gamma \delta} \zeta_{\gamma} \zeta_{\delta}}{\zeta_{1}^{2}}
$$

and the 4 th order polynomial $f(\omega)$ by

$$
f(\omega)=\frac{\Lambda_{\alpha \beta \gamma \delta} \zeta_{\alpha} \zeta_{\beta} \zeta_{\gamma} \zeta_{\delta}}{\Lambda_{2222} \zeta_{1}^{4}}
$$

where $\zeta\left(\zeta_{1}, \zeta_{2}\right)$ is the Fourier transform domain vector and $\omega=\zeta_{2} / \zeta_{1}$. Using the above definitions and complex number notation, the fundamental solution can be written in the following compact form

$$
u_{\alpha \beta}^{*}(\mathbf{x} \xi \quad)=-\frac{1}{2 \pi \Lambda_{2222}} \operatorname{Re}\left(\sum_{\kappa=1}^{2} \frac{F_{\alpha \beta}\left(\omega_{\kappa}\right)}{\beta_{\kappa}} \ln v_{\kappa}\right)
$$

where

$$
\begin{gathered}
v_{\kappa}=x_{1}-\xi_{1}+\omega_{\kappa}\left(x_{2}-\xi_{2}\right) \\
\beta_{1}=b_{1}\left(\omega_{1}-\omega_{2}\right)\left(\omega_{1}-\bar{\omega}_{2}\right) \\
\beta_{2}=b_{2}\left(\omega_{1}-\omega_{2}\right)\left(\bar{\omega}_{1}-\omega_{2}\right) \\
\omega_{\kappa}=a_{\kappa} \pm \mathrm{i} b_{\kappa},\left(\kappa=1,2 ; b_{\kappa}>0\right)
\end{gathered}
$$

are the complex roots of $f(\omega)=0$

and $\bar{\omega}_{\kappa}$ are the complex conjugates of $\omega_{\kappa}$. The fundamental strains are obtained by combining the deformation gradients

$$
u_{\alpha \beta, \gamma}^{*}(\mathbf{x} \xi)=-\frac{1}{2 \pi \Lambda_{2222}} \operatorname{Re}\left[\sum_{\kappa=1}^{2} \frac{F_{\alpha \beta}\left(\omega_{\kappa}\right)}{\beta_{\kappa} v_{\kappa}}\left(\delta_{1 \gamma}+\omega_{\kappa} \delta_{2 \gamma}\right)\right]
$$

The first fundamental solution of the plate bending problem is given by [5]

$$
w_{1}^{*}(\mathbf{x} \xi)=w^{*}(\xi)=\frac{1}{4 \pi D_{2222}} \operatorname{Re}\left(\sum_{\kappa=1}^{2} \frac{r_{\kappa}^{2}}{\gamma_{\kappa}} \ln r_{\kappa}\right)
$$

where

$$
\begin{aligned}
& r_{\kappa}=x_{1}-\xi_{1}+\omega_{\kappa}\left(x_{2}-\xi_{2}\right) \\
& \gamma_{1}=h_{1}\left(\omega_{1}-\omega_{2}\right)\left(\omega_{1}-\bar{\omega}_{2}\right)
\end{aligned}
$$




$$
\begin{gathered}
\gamma_{2}=h_{2}\left(\omega_{1}-\omega_{2}\right)\left(\bar{\omega}_{1}-\omega_{2}\right) \\
\omega_{\kappa}=g_{\kappa} \pm \mathrm{i} h_{\kappa},\left(\kappa=1,2 ; b_{\kappa}>0\right)
\end{gathered}
$$

are the complex roots of $f^{N}(\omega)=\frac{D_{\alpha \beta \gamma \delta} \zeta_{\alpha} \zeta_{\beta} \zeta_{\gamma} \zeta_{\delta}}{D_{2222} \zeta_{1}^{4}}=0$

The fundamental curvatures are thus obtained as

$$
w^{*},_{\alpha \beta}(\mathbf{x} \xi)=\frac{1}{4 \pi D_{2222}} \operatorname{Re}\left[\sum_{\kappa=1}^{2} \frac{2 \ln r_{\kappa}+3}{\gamma_{\kappa}}\left(\delta_{1 \alpha}+r_{\kappa} \delta_{2 \alpha}\right)\left(\delta_{1 \beta}+r_{\kappa} \delta_{2 \beta}\right)\right]
$$

\section{References}

[1] Whitney, J.M. and Leissa, A.W., Analysis of heterogeneous anisotropic plate. Transactions ASME, Journal of Applied Mechanics, 36, pp. 261-266, 1969.

[2] Whitney, J.M. and Leissa, A.W., Analysis of a simply supported laminated rectangular plate. AIAA Journal, 1(8), pp. 28-33, 1970.

[3] Whitney, J.M., The effect of boundary conditions on the response of laminated composites. Journal of Composite Materials, 2(4), pp. 192-203, 1970.

[4] Harris, G.Z., Buckling and post-buckling behaviour of composite plates under biaxial loading. International Journal of Mechanical Sciences, 17(3), pp. 187-202, 1975.

[5] Syngellakis, S. and Cherukunnath, N., Boundary element analysis of symmetrically laminated plates. Engineering Analysis with Boundary Elements, 28(9), pp. 1005-1016, 2004.

[6] Hwu, C., Boundary integral equations for general laminated plates with coupled stretching-bending deformation. Proceedings of the Royal Society of London, Series A (Mathematical, Physical and Engineering Sciences), 466(2116), 2010.

[7] Shi, G. and Bezine, G., Buckling analysis of orthotropic plates by boundary element method. Mechanics Research Communications, 17(1), pp. 1-8, 1990.

[8] Syngellakis, S. and Cherukunnath, N., Stability analysis of laminate plates by the boundary element method. Boundary Elements XXIV, ed. C.A. Brebbia, A. Tadeu and V. Popov, WIT Press: Southampton, pp. 197-206, 2002.

[9] Cherukunnath, N. and Syngellakis, S., A boundary element approach to buckling of laminated plates subjected to arbitrary in-plane loading. 43rd AIAA/ASME/ASCE/AHS/ASC Structures, Structural Dynamics and Materials Conference, Papers-AIAA: pp. 866-874, 2002.

[10] Syngellakis, S. and Cherukunnath, N., Boundary element modelling of nonlinear buckling for symmetrically laminated plates. Mesh Reduction Methods BEM MRM XXXI, ed. C.A. Brebbia, WIT Press: Southampton, pp. 211-222, 2009. 
[11] Syngellakis, S., A boundary element approach to buckling of general laminates. Boundary Elements and other Mesh Reduction Methods XXXIV, ed. C.A. Brebbia and D. Poljak, WIT Press: Southampton, pp. 145-155, 2012.

[12] Timoshenko, S.P. and Woinowsky-Kreiger, S., Theory of Plates and Shells, McGraw-Hill: New York, 1964.

[13] Rizzo, F.J. and Shippy, D.J., A method for stress determination in plane anisotropic elastic bodies. Journal of Composite Materials, 4, pp. 36-61, 1970.

[14] Shi, G. and Bezine, G., A general boundary integral formulation for the anisotropic plate bending problems. Journal of Composite Materials, 22, pp. 694-716, 1988.

[15] Perez, M.M. and Wrobel, L.C., An integral equation formulation for anisotropic elastostatics. Journal of Applied Mechanics-Transactions of the ASME, 63(4), pp. 891-902, 1996.

[16] Bui, H.D., Some remarks about the formulation of three-dimensional thermoelastoplastic problems by integral equations. International Journal of Solids and Structures, 14(11), pp. 935-939, 1978.

[17] Syngellakis, S. and Wu, J., Nonlinear viscoelastic fracture mechanics using boundary elements. Key Engineering Materials, 454, pp. 137-148, 2011.

[18] Budiansky, B., Theory of buckling and post-buckling behavior of elastic structures. Advances in Applied Mechanics, ed. C.S. Yih, Academic Press: New York, pp. 1-65, 1974. 